\title{
Computer Vision on a Parking Management and Vehicle Inventory System
}

\author{
Aaron Don M. Africa, Antonio Miguel Sarmiento Alejo, Grant Lewis Milan Bulaong \\ Samantha Maxine Ronquillo Santos, Jerrick Spencer Kehyeng Uy \\ De La Salle University, Manila \\ 2401 Taft Ave., Malate, Manila 1004, \\ Philippines, aaron.africa@dlsu.edu.ph
}

\begin{abstract}
Over time, scientists and engineers have developed technology that has improved everyday living. For every invention, they have found to reinvent it and create a better version of it. An example would be parking lots. From the usual time-in-time-out system; window cashier to parking spot back to window cashier, the hassle of this cycle was reduced through the use of license plate recognition which this study tackled. License plate identification technology has become a useful factor in different issues like parking management, vehicle owner recognition, etc. Computer vision has provided a means to reduce manual labor in parking lots by replacing them with sensors and scanners which would take over. This would be beneficial to the company as this technology would require minimal maintenance. Through image processing and enhancement, the license plates of these cars could be taken account of in real-time and this data could be used to generate the parking bills given the time-in and a time-out of the vehicle. This paper aimed to tackle the problems of car management and to further simplify the parking system.
\end{abstract}

Key words: License Plate Recognition, Parking management, Sensors, Time-in Time-Out System.

\section{INTRODUCTION}

Current parking schemes found in malls and other public use parking spaces operate a vehicle counting systems that can track the volume of free space available in a lot. These schemes although allows the driver to get information on what area to park lacks the ability to convey which specific parking space is free. This results in cars roaming around in an area to locate the mentioned parking spot. This causes cars to congest in certain areas in parking spaces as well as slows down the segregation of cars. With the use of computer vision, the cars about to enter the parking lot could be accounted for and possibly allocated to a specific free parking space. Computer vision enables the use of images and high-dimensional data to form numerical or symbolic data that can accurately inspect and read the information, being able to use and apply this information in different tasks such as automation, data gathering and decoding of data [1]. In addition, this task is a close recreation of what the human vision can do by perceiving and understanding the data given to the program.
One approach in recognizing the license plate number there are three phases in achieving this task, image capturing, plate recognition and number recognition [2]. The first phase, image capturing processes the image by filtering out the color of the image separating the RGB spectrum and generating a grey scale image. The second phase, plate recognition makes use of the different filtered images to scan for differences in the frequency bands of the image. The license plate could be located this way since the license plate has a higher frequency, hence by using gaussian filter the vertical frequency as well as the horizontal frequency could create a pixel intensity graph. Lastly the final phase, number recognition converts the gray scaled plate number into a black and white image and using a character matching algorithm, identify the letters depending on the location of the symbols on the plate number. Using computer vision this is one of the possible processes that could be done to analyze the license plate.

\section{BACKGROUND OF THE STUDY}

Digital Communications has been utilized in order to transmit data through a digital form. The data that is transmitted could vary from one topic from another. The researchers have decided to delve on the topic of Computer Vision in Parking Management. Research has been made previously about traffic enforcement, but this study focused on parking specifically. This research utilized spatial imaging, rough set theory, electronic sensors, logic scoring of preference, artificial neural network, database monitoring, and data or information transfer. All of which were tackled in digital communications. These features were subcategorized in the different parts of the research. Spatial imaging and Artificial Neural Network were under license plate detection. Rough Set Theory, Logic Scoring of Preference, Database Monitoring and Data or Information Transfer were all under Time-based monitoring system. Lastly, electronic sensors would be tackled through the sensors for detecting parking. The basic flow of the system goes as follows: the car that was parked would be detected by sensors, and the license plate would then be taken note of through character recognition. The image was then enhanced through image processing so that the software would be able to recognize the digits of the plate number. The digits were then stored into a database and would be pulled out when it's needed. The research could be used for maintaining a parking space without having people in it. This would reduce the need for human labor and would allow more 
efficient processing. Through the implementation of this system in parking spots around the country, parking bills could be generated almost immediately. The time-in and time-out of the cars would be monitored and the price of the parking bill with respect to the time could be accounted for. For this specific study, the researchers used simulation in order to analyze the system.

\section{A. Spatial Imaging}

This feature was highlighted through the system's usage of an image processing algorithm using computer vision to locate the digits of the parking license and to provide the driver with their own parking spot.

\section{B. Rough Set Theory}

This feature allowed the data to be categorized into different compartments in the database for easier access in the future. An example of this would be the car built. The size of the car would be taken into account by the system and would provide them the necessary parking spot.

\section{Electronic Sensors}

In order for the system to function, sensors were placed to be able to obtain the data. A camera was used per parking spot to be able to capture the image of the license plate and the cars so that the system would know how many more spaces were available.

\section{Logic Scoring}

This feature improved the decision-making algorithm of the system. This was done by having weighted variables when encoding and segregating the car system.

\section{E. Artificial Neural Network}

This feature was used in managing the cars respectively given the available parking spots.

\section{F. Database Monitoring}

The database monitoring was used to keep the data in the system that would be used later on. It was also used for the processing of the data of the license plates in order to generate their electric bills as well as the reading of the characters in the license plate.

\section{G. Data/Information Transfer}

This feature was utilized from the transmitter to the receiver through the sensors. The data that was obtained was the digits of the license plate that would be stored in the database.

\section{STATEMENT OF THE PROBLEM}

It is arguable that commercial parking spaces today can be made to operate more efficiently. It is a common experience that during busy hours, drivers take several minutes attempting to locate a free parking spot while driving around the car park [3]. Parking management and vehicle inventory system will help to resolve the growing problem of wasted time, traffic congestion, and help provide better service, reduce car emissions and pollution, improve city visitor experience, increase parking utilization, and prevent unnecessary capital investments [4]. An automatic parking management system such as that which is proposed in this paper can do this by utilizing an image recognition processor and a central computer to automatically assign a parking space to a vehicle that enters the park. This can be done through cameras and sensors at the entrance of the park as well as throughout each of the parking spaces. A computer system manages the whole process and controls various display panels and lights that help the driver in parking his car.

\section{SIGNIFICANCE OF THE STUDY}

The study proved to be significant due to, the most evident problems, traffic congestion, and inefficient parking management. In the everyday lives of motorists, they have been victims of stress and wasted time as an effect of long waiting queues just in search of a parking lot. However, it was not just those looking to park who were affected by the poor and inefficient conventional parking lot systems. Day to day motorists just passing by a certain area with a high volume of vehicles waiting to be parked have also been negatively affected due to the consequent traffic congestion. Additionally, traffic congestions in highly urbanized areas have proven to produce an increase in air pollution in their respective areas. Furthermore, in a fast-paced modernized world, new and improved technology have not been maximized especially in terms of parking management. Tracking via computer vision has developed continuously and consequently needs to be implemented in day to day systems. With all of those in mind, the researchers came to the conclusion that their study must revolve around computer vision for parking management and vehicle inventory systems. With the use of tracking via computer vision of license plates and other possible vehicle identifiers, managements may have possibly been able to effectively monitor and systematically disseminate proper information regarding their parking space availabilities.

\section{DESCRIPTION OF THE SYSTEM}

The proposed system utilizes a plate recognition processor and a central computer to automatically assign a parking spot to a vehicle that enters the park [5]. The use of an image recognition unit at the entrance of the parking area allows for the identification and tracking of the vehicle within the park through its plate. A computer manages the occupancy of the park by detecting if assigned vehicles to corresponding parking spots are correctly placed. This is checked by the use of the same plate recognition system at each of the parking spots. 


\section{METHODOLOGY}

An existing image recognition system was used to log plates at the entrance of the parking area. The system processes images taken from a camera at the car park entrance to extract the licensed plates of any vehicle entering. Fig. 1 showed the block diagram of the system.

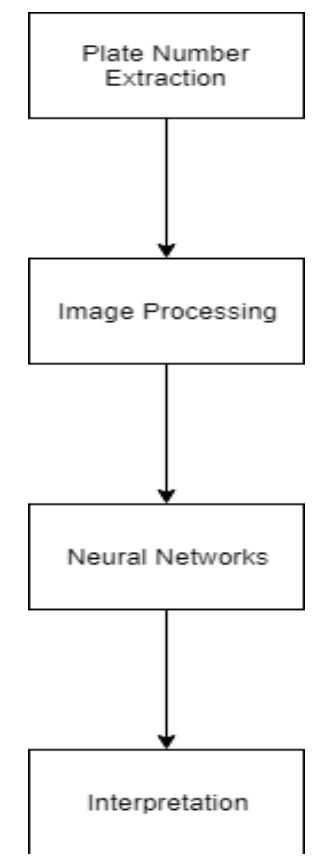

Figure 1: Block Diagram of the Plate Recognition System

First, the plate number is extracted by taking an image using a camera. Then, the captured image is digitally pre-processed to remove skewness and to segment characters from each unit of the image. This is the input fed to an Artificial Neural Network (ANN) for training, validation, and testing, after which the plate is decoded and interpreted.

Next, the image recognition system sends the license plate information to a central computer where a parking spot is assigned to the vehicle. A camera located at the dock of each parking spot utilizes the aforementioned image recognition system to verify that the vehicle parked is indeed the vehicle that is assigned to the spot. If the verification succeeds, then the central computer system is notified and the parking time for the vehicle is recorded. This may be used to apply variable pricing methods on the utilization of the space. If the verification fails, then the system sounds a short-lasting alarm, and the license plate is tagged in the central system if the vehicle does not leave the parking spot in a short period of time. This may be used to impose penalties for management.

As a means of testing the effectiveness of the parking management system against traditional methods, the time for a vehicle to locate a parking spot under the system will be measured and compared to that which is obtained in a parking space without such system.

\section{REVIEW OF RELATED LITERATURE}

\section{A. Computer Vision}

There were three individual and critical entities for the research conducted [6]. The first entity was the computer vision, the second entity was the processing of images, and finally, the last entity was the extraction of image features. Although the entities mentioned were separated, they were still somewhat interconnected. They were interconnected in a way because, in order for one entity to function, another entity was needed. More specifically, in order for the second entity, image processing, to be done and analyzed successfully, the computer was needed. Consequently, it would have then been used to distinguish unchanging qualities and information from the subject. Finally, with all the data gathered from the initial steps of image processing, they would have been utilized to sort through and manage specific challenges. Moreover, with the numerous researches conducted on technology for image processing, its most basic principles concluded were utilized as a guide for the researchers in their analysis of applying computer vision technology in diverse disciplines [7]. Additionally, those concluded principles were also used as a basis for the researchers to carry out their analysis of the difference between methods.

In order to carry out their research focused on computer vision, two types of equipment were critical in the process. The first type of equipment involved was for imaging and the latter was digital [8]. One of the main reasons behind their experiment of computer vision was to allow computers to function in a similar way as human beings do. In that fashion, computers were to be modified and programmed to be able to not only observe but also comprehend and adapt to their surroundings through their garnered vision. It was with that in mind that the researchers also wanted the computer to be able to do all those autonomously. In an fast paced technological world where everyone was connected through the web, and living wirelessly even with the cloud, the ability to go through specific image data was of paramount importance. Even with the large and scattered database of images, the researchers wanted to be able to identify and logically connect certain networks of information [9]. They believed that in doing so, they would be able to predict social trends and behaviors.

Digital image processing would not have reached the excellence it was known for if not for deep learning [10]. Deep learning went beyond what was expected when it came to the whole processing. Even so, computer vision methods, which arrived earlier than deep learning, have shown continuous progress in terms of advancements in the 
field of digital image processing. It was with those in mind that the researchers decided to analyze the pros and cons of the two processes. They aimed to determine and motivate discussions regarding the importance of maintaining computer vision techniques and pursuing its development. Aside from the pros and cons, they were able to identify the capability of intertwining both deep learning and computer vision methods. Moreover, it was with the discussions and researches that they realized certain problems were more manageable with computer vision and almost impossible with deep learning [11]. Even so, they managed to determine the hybrid capabilities of certain technology such as 3D and Panoramic Vision. It was in those types of technology that the researchers realized the importance of allowing computer vision methods to work with deep learning for all models to be maximized.

The drivers and motorists were highly affected by the availability of parking slots [12]. A systematic approach to monitor said slots would have been an effective way for users to efficiently manage their schedules and expenses. Consequently, the researchers emphasized that a more efficient parking system for the motorists were also affecting the rest of the community positively as it reduced air pollution in the area. It was with those in mind that the researchers aimed to focus on developing a computer vision system capable of managing and calculating for free parking slots within a designated area. In order for them to manage and calculate the free slots, they decided to rely on three estimation methods. Concisely, their plan was to analyze the widest area covered in a video frame, therefore having the highest number of similarly colored pixels, and set it as background or the designated area for parking cars. The collected results were then narrowed down for the final estimated count using a Multi-Layer Perceptron [13]. Moreover, in order to verify their data, they compared their count with the count collected from payment registers to determine the accuracy. It was even tested in Warsaw and resulted in an accuracy of $95 \%$.

Computer vision presented a safe way to possibly implement self-driving vehicles [14]. Based on recent amounts of research, the number one threat of having self-driving vehicles on public roads was the fact that they might cause accidents or even deaths in terms of human lives. It was always with that in mind that the topic of self-driving cars was very much debated with heavy arguments. Officials focused on public safety, policies, and technology have argued about the topic endlessly. A critical example against the proposal was the accident involving Uber Incorporated as their self-driving car caused the death of someone walking the street it crashed in. It was with that one incident that numerous policymakers have voted against allowing said vehicles to be utilized on public roads. Consequently, the researchers aimed to focus on the possibility of avoiding the crash by utilizing computer vision models [15]. Their models were capable of analyzing numerous techniques for enhancing and recognizing certain images and objects in varying amounts of light. With the analysis, the safety of pedestrians was ensured even in conditions with low amounts of light.

Analysis and mapping of parking locations through aerial imaging was possible [16]. Through an algorithm for pattern recognition implemented with computer vision, the said processes were achievable. Their research was split into two parts namely analysis and mapping. In terms of the former part, the researchers utilized line detection processes highly sensitive to selectivity. The lines were selected depending on properties such as lengths and angles. With their algorithm, they wanted to be able to detect automatically the volume and availabilities in certain areas of a parking space. They utilized a quad-copter with a camera on a gimbal for parallel-angled aerial video frames and images to be collected then consequently selected. Those images were vital to the implementation and testing of their algorithm. Afterward, the gray-scaled the images and applied a binary filter to make line features more distinct for the Hough transform. Through numerous iterations, they determined the repetitive line patterns and filtered out the noise in order to group the aligned edges for single parking slots.

Facial landmarking systems all over the world were being developed due to computer vision [17]. Computer vision was a basis for all facial processing techniques as it went from the capability to recognize not only faces but emotions as well. A modern example of the system was seen in the social networking site, Facebook. It allowed for the identification and tagging of specific people in photos automatically. A few other applications were also capable of initially identifying a user's emotion then consequently providing a similar emoticon. On a higher level, facial landmarking systems were seen to be highly effective in terms of security applications. Specifically, for their research, they utilized computer vision techniques and combined it with algorithms for deep learning in order to develop their facial keypoint recognition, more commonly known as those specific points around distinguishing facial features like the eyes, mouth, and nose. Initially, they pre-processed images and detected faces. Afterward, they trained a neural network to detect distinguishing facial features.

Travelers with eyesight problems were usually most at risk when it came to crossing intersections [18]. No intersection was ever the same due to varying traffic and locations. It was due to those facts that planning for dynamic environments required devices like canes and talking signals to navigate through intersections. The researchers, therefore, decided to focus on developing a computer vision-based system capable of giving the visually impaired user accurate guidance when it came to intersections. The initially worked on the real-time signal 
detection in order to recognize whether the pedestrian may or may not cross with respect to the information given off by traffic lights. Mobile visual computing along with cognitive assistance allowed for verbal guidance when navigating through real-time intersections.

The researchers focused on city traffic controlling with the use of proposed methods and algorithms effective for analyzing visual data from transportation monitoring systems [19]. In order to maximize the implementation, public transportation vehicles need to be recognized and mapped with the camera. In their research, they discerned the structure and collected their data using their algorithm capable of removing the background of a moving vehicle within a certain scene. Their system then classified the vehicles and rejected those which were not city buses. Consequently, they were able to develop a specific tracking system for labeling city buses.

In order for autonomous driving to fully be optimized, a system capable of finding lanes should have first been developed [20]. The researchers applied computer vision to allow for finding lanes on certain roads and motorways. Their system basically transformed their captured images into binary data then consequently applied a dynamic histogram window in order to pinpoint the exact position of the lane markers. They were able to develop their system to function in real-time accurately.

\section{B. Parking Management}

The researchers utilized a wireless sensor network, with both a microprocessor and app in parking lots with smart guides [21]. Their creation was an improved version of another one previously created [22]. They mentioned that current surveys showed only prompts and remaining parking locations when it came to a lot of management. Due to the increasing number of vehicles, economic development, and urbanization, the motorist has been wasting a large amount of their time simply looking for available parking areas. With that in mind, the researchers wanted to integrate a smart function with an internet of things system development feature. Their project aimed to provide self-managed parking through mobile devices to find the closest parking area and even view the number of available slots. It was even capable of guiding the driver to the available slot as soon as he/she entered the parking lot area. Finally, they were able to develop their program to provide the number of hours parked along with the corresponding parking fee per area.

A system was created in a built environment for transport management in certain smart cities [23]. It was mentioned that while motorists waste their time in search of parking, their congested volume consequently caused air pollution [24]. It was with that in mind that they created their Intelligent Parking Information Systems capable of making the dissemination of real-time parking vacancies more efficient through applications in smartphones. They discovered that downloading habits, parking time, and parking application usage were the three most positive impacts on the consumers' willingness to pay for applications involving similar smart systems.

Street parking availability has proven itself to be a critical part of an urban transportation system [25]. Their creation was similar to another one already created but with improved features [26]. Regardless of how fast businesses spread, the central business districts still and always will attract numerous customers who had little to no choice but to park on the streets. Due to those vehicles parked, and without a specific on-street parking policy, the situation has shown to be quite chaotic. It was with that in mind that the researchers decided to create a system to assess on-street parking demand rationally to manage to park. They were able to capture all variations of the parking demand and analyze numerous ways to carry out parking inventory and evaluation for designated intervals in commercial areas. By using data monitoring, they were able to facilitate plans and policies made for on-street parking management measures for developing countries.

Ultimate parking problems in urban areas could have been solved by the internet of things based traffic and routing management system [27]. Further understanding of traffic and routing management systems allowed them to develop specific features of their internet of things solution [28]. Even with the continuous increase in vehicular volume, the researchers aimed to allow motorists to locate vacant parking spaces in order to save on time and fuel. Through their application, the user was able to view the systematic count of the available capacity in terms of parking spaces and was consequently notified when a parking space freed up. Their system consisted of an ultrasonic sensor to detect the data needed by the microcontroller which consequently updated the cloud server for logging data. Their system allowed users to manage their time in parking areas by allowing them to view slots earlier.

Vehicles have constantly been updated to contain computers and communication devices in order to connect applications and services in the field [29]. Vehicular ad hoc networks were still in progress as they were capable of possibly exchanging data between vehicles, applications, services, and sources [30]. The researchers created a crowdsourcing scheme for the data gathering in a specified area depending on their environment, atmospheric qualities, and even parking space availabilities. Their method allowed for mobile technology to complete and disseminate data among vehicles from the same classifications.

Most researchers have been focusing on vehicle detection through magnetic sensors [31]. In terms of smart parking management systems, magnetic sensors or wireless vehicle 
detectors simply utilized low power wireless communication technology for the convenience of data transfer and hardware maintenance. Even so, those magnetic sensors have proven some instances of failure due to adjacent vehicles and dead zones, which ultimately reduced the accuracy of detection. With that in mind, the researchers created an RF-based vehicle detection system depending on received signal strengths from wireless transceivers. Their system was made only to function when the magnetic signals were insufficient to provide accuracy as the wireless transceivers were more demanding in terms of energy.

Car plate recognition has proven to be useful in terms of parking lot management, security, tolls, traffic monitoring, and so on [32]. Normally in surveillance systems, the output was usually low in resolution to the point of the text is unreadable. It was with this in mind that the researchers utilized super-resolution methods to improve the quality by still using the low-resolution images and processing them into a high-resolution image. They also managed to solve quality and smoothing issues through perceptual loss. Their output quality was to be compared to existing bicubic techniques.

Mobile edge computing was capable of creating an intelligent parking management system [33]. The researchers were able to detect vehicles, bind plates, and upload information. They utilized magnetic sensor and gateway edges and consequently designed a way to reduce power consumption for devices with limited power. They were able to initially map the management system onto a mobile edge computing model and consequently converted the offload onto optimization issues. By doing so, they were able to improve the system's overall efficiency, power dissipation, transmission cost, and latency.

Smart mobility was of paramount importance in developing nations worldwide [34]. With the fast-paced economic lifestyle, double-parking, and busy roadside activities from deliveries have proven to be a problem. The researchers, therefore, created a real-time internet of things-based system to monitor roadside loading and unloading bays. They utilized high definition smart cameras integrated with wireless communication systems for traffic surveillance. Since they used a vision-based network, real-time images were captured autonomously. Through the collected data, roadside vacancies were able to be analyzed with fuzzy logic and even showed to users for transparency.

Through mathematical programs with equilibrium constraints, they were able to pinpoint the best street parking facilities within a work area [35]. With the best street parking site facilities, the network would have had the shortest waiting time. The researchers created a variational inequality to simulate the single response equilibrium for parkers and logit-based stochastic user equilibrium for travelers just passing through. The initial allowed for all possible routes in the shortest amount of time delay. They were also able to solve the same problem through iterations in their radial basis function.

\section{THEORETICAL CONSIDERATIONS}

The system demands the utmost accuracy (highest success rate) for plate recognition for images taken from both the entrance camera and each of the parking spot cameras. Should an incorrect mismatch occur on a vehicle plate; a false success may occur, thus charging the incorrect vehicle; or a false failure may occur, causing the incorrect imposition of penalties. To minimize errors within the digital communication system, identical cameras must be used. A character recognition accuracy of $92 \%$ has already been achieved [36]. On the other hand, another system has also achieved $97 \%$ [37]. The image recognition system to be used obtained an accuracy of $99.98 \%$ for 5,680 sets of training data.

The plate recognition unit utilizes a multi-layered ANN with training data which may not closely match the processed inputs fed into by the camera. Thus, it is necessary to retrain the neural network to fit our new set of data [38]. Furthermore, the input delay of the neural network must be accounted for as the goal of the system is to produce results in real-time.

\section{DATA AND RESULTS}

Simulations have already been created that would produce an error percentage of 0 . $00000004 \%$ which is generally very small. The success rate of image recognition has already been found to be almost $92 \%$ to $97 \%$ which is generally almost error-free. Given that previous image, systems have already reached a $99.98 \%$ accuracy, it could reach a perfect percentage of a hundred if the training data were to be increased.

A person may take 5 to 10 minutes just looking for a parking spot without it being guaranteed that they would be able to find one. This issue could be resolved through the system because it would be able to provide the driver that is about to enter the parking spot with a list of parking spaces that could be used. The hassle of looking for a parking spot would be eliminated through the system because it would also be able to tell them if there is no more space left. Given that in previous research, the system was able to reduce traffic inside parking spaces by almost $30 \%$.

Additionally, the MathWorks website has provided a Vehicle Number Plate Recognition algorithm that utilized a template matching technique. A vehicle number plate recognition algorithm that integrated image detection and processing has already been created [39]. He first created the template using the code in Fig. 2. Afterward, Fig. 3 was the creation of a letter and number matrix. Fig. 4 was created for the letter detection of the software. Fig. 5 was written for plate detection. Fig. 6 was used to detect the location of the plate in the image that was processed. Fig. 7 was used for image clarification. Using the functions imcrop and bwareaopen, it is able to crop the number and remove unnecessary noise from the image. 
Aaron Don M. Africa et al., International Journal of Emerging Trends in Engineering Research, 8(2), February 2020, 323 - 332

\section{\%Alphabets}

A=imread('alpha/A.bmp');B=imread('alpha/B.bmp');(=imread('alpha/C.bmp'); $D=$ imread('alpha/D. bmp') ;E=imread('alpha/E.bmp');F=imread('alpha/F.bmp'); G=imread('alpha/G.bmp');H=imread('alpha/H.bmp');I=imread('alpha/I.bmp'); J=imread('alpha/J.bmp');K=imread('alpha/K. bmp'); i=imread('alpha/L.bmp'); M=imread('alpha/M. bmp');N=imread('alpha/N. bmp');0=imread('alpha/0. bmp'); $P=i m r e a d((' a l p h a / P . b m p ') ; Q=i m r e a d(' a l p h a / Q . b m p ') ; R=i m r e a d(' a l p h a / R . b m p ') ;$ S=imread('alpha/S.bmp');T=imread('alpha/T.bmp');U=imread('alpha/U.bmp'); $V=i m r e a d($ ('alpha/V.bmp'); ; =imread('alpha/W. bmp');X=imread('alpha/X.bmp'); $Y=i m r e a d($ 'alpha/Y.bmp');Z=imread('alpha/Z.bmp');

\%atural Numbers

one=imread('alpha/1.bmp'); two=imread('alpha/2.bmp');

three=imread('alpha/3.bmp'); four=imread('alpha/4.bmp');

five=imread('alpha/5.bmp'); six=imread('alpha/6.bmp');

seven=imread('alpha/7.bmp'); eight=imread('alpha/8.bmp');

nine=imread('alpha/9.bmp'); zero=imread('alpha/0.bmp');

\%(reating Array for Alphabets

letter=[A B C D E F G H I J K L M N O P Q R S T U V W X Y Z];

\%(reating Array for Numbers

number=[one two three four five six seven eight nine zero];

NewTemplates=[letter number];

save ('NewTemplates', 'NewTemplates')

clear all

\section{Figure 2: Code Part A}

Xcreating Array for Alphabets

lettera[ $A$ B C D E F G H I ] KL M NOP Q R S T U V W X Y Z];

XCreating Array for Numbers

nurbera[one two three four five six seven eight nine zero];

NewTemplates-[letter number];

save ('NewTeaplates', 'Newiemplates')

clear all

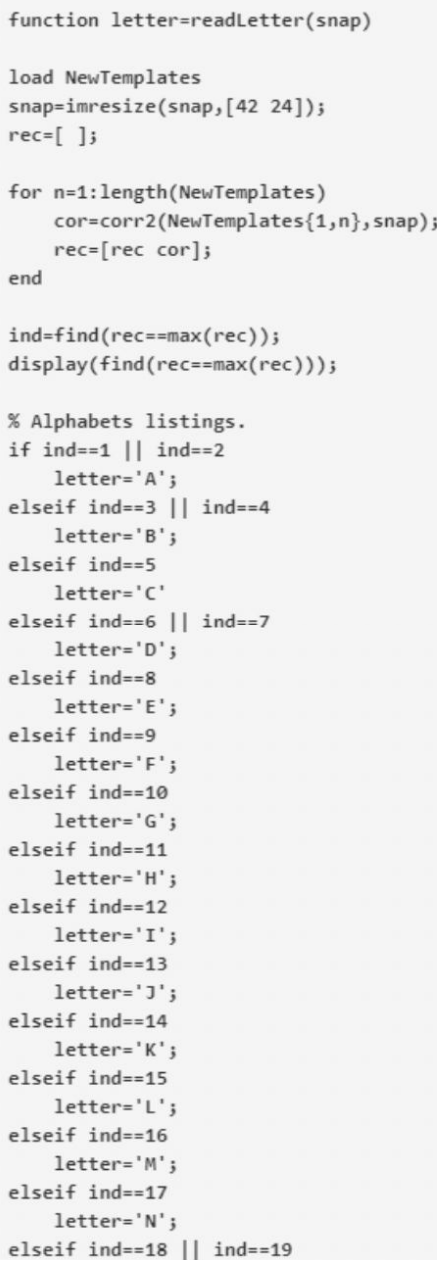

Figure 4: Code Part C

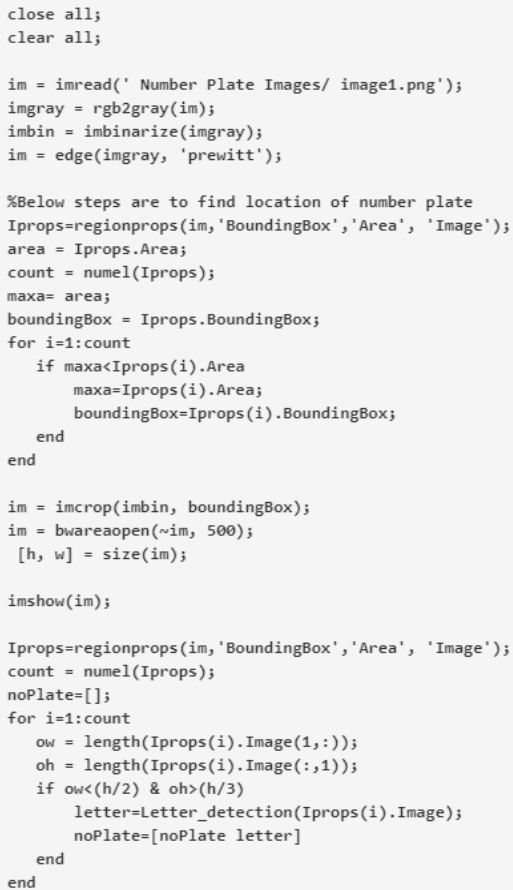

Figure 5: Code Part D lseif ind $=20$. ind $=21$ letter $=$ ' $P$ '; lseif ind $==22$ || ind $==23$

letter=' $Q$ ';

elseif ind $=24$ || ind $=25$ letter $=$ ' $R^{\prime}$;

elseif ind $=26$

letter=' $S$ ';

elseif ind $=27$

letter $=$ ' $T$ ';

elseif ind $=28$

letter = ' $U$ ';

elseif ind $=29$

letter $=$ ' $V$ ';

elseif ind $=30$

letter=' $W$ ';

elseif ind $=31$ letter=' $X$ ';

elseif ind $==32$

letter $=$ ' $Y$ ';

elseif ind $=33$

letter $=' Z$ ';

\%*-*_*_*_*

\% Numerals listings.

elseif ind $==34$

letter $=1{ }^{\prime}$;

elseif ind $==35$

letter $=$ ' 2 ';

elseif ind $=36$

letter $=$ ' 3 ';

elseif ind $==37$ || ind $==38$

letter $=4^{\prime}$;

elseif ind $==39$

letter $=5^{\prime}$;

elseif ind $=40$ || ind $==41$ || ind $=42$

letter $={ }^{\prime} 6^{\prime}$;

elseif ind $=43$

letter $=$ ' 7 ';

elseif ind $=44$ || ind $==45$

letter $=^{\prime} 8^{\prime}$;

elseif ind $=46$ || ind $==47$ || ind $==48$

else

letter $={ }^{\prime}$

letter $={ }^{\prime} \theta$;

end 


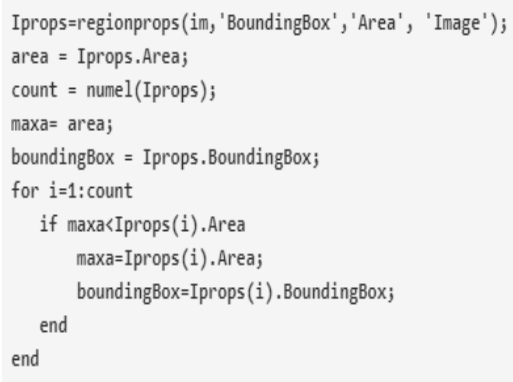

Figure 6: Code Part E

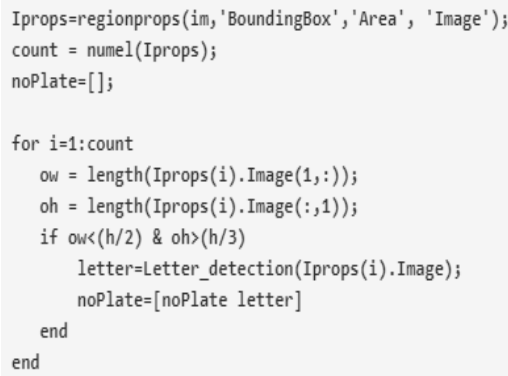

Figure 7: Code Part F

\section{ANALYSIS OF DATA}

Subject to the practical accuracy of the results, the probability that a mismatch occurred between the plate recognition system pairs between the parking entrance and one of the parking spots is $0.00000004 \%$. Although this may already be deemed a highly efficient method, this error probability may be further optimized and reduced by training the ANN with new data obtained from the cameras of the system. This requires the manual reporting and tagging of incorrect plate numbers, should any arise. Also, the plate recognition system proposed was found to generate the license plate numbers within three seconds, thus it is appropriate for achieving real-time results in parking management.

Since the system is capable of tracking the time-of-use of the parking spots, managers of the space were able to control billing for each individual car, penalties for misplaced cars as well as manage the time-of-use of each spot. No vehicle assignment errors were found when the digital system was implemented. One problem encountered by the system is that the cameras used for each individual parking spot may be impeded by the user to avoid time-tracking, either intentionally or by using what is known as an adversarial patch $[40,41]$. This interferes with the system because the controlling computer loses track of the vehicle within the vicinity of the parking space. For this error the Rough Set Theory can be used [42]. This causes an apparent 'mismatch' between plates resulting in an error within the digital communication system.

Finally, the proposed parking management system effectively reduced the time taken for a car to park within the space by eliminating the 'hunting' process completely. Users of the system found that they no longer have to check individual floors for available parking because the use of assigned parking spots assisted them through the entire process. This system will use the databases in this study $[43,44,45,46]$. The proposed system was found to reduce internal traffic moving times by up to $30 \%$.

\section{CONCLUSION}

In conclusion, with the application of a car park network system, the operation of the segregation system increases the efficiency of the car park as well as improves the experience of parking. The amount of time that the drivers take in finding a parking spot decreases since specific parking spots would already be pinpointed for the drivers. With the use of different image processing tools, this could be possible through the use of computer vision or an artificial neural network. The steps to execute this network used a plate number extraction processor, followed by the image processor that would decode the number found on the license plate. The neural network then segregates the cars and allocating them to specific parking spots. Since the parking spots would already be allocated to the driver this decrease traffic congestion in the parking lot as well as improved parking utilization; while also creating a space that would allow a free flow of traffic in the parking lots. In addition, the use of this system would increase the experience for city visitor and increase the utilization of the parking space. Aside from this feat, the network system would also increase safety in the parking lot since fewer cars would roam. With the use of computer vision, this system manages the whole process itself without the supervision of a person handling the system. The use of sensors and different light displays would also allow the drivers a better grasp of where to go in the parking lot. In summary with this proposed network system parking lot management would perform more efficiently while also affecting different factors outside of the parking lot; some examples referenced were parking lot lines found congesting areas in the parking lot as well as outside the parking lot. On the other hand, since the system operates without the help of human management this system would be fully autonomous creating a self-managing efficient parking system.

\section{RECOMMENDATIONS}

The researchers highly recommend future analysts to find a means to create a simulation on the study on parking management through a software. With the equipment that the researchers currently have, they found it difficult to create a simulation that would be able to convey all the steps in the computer vision system. Another recommendation would be to research further into past studies on parking management through the use of computer vision in everyday products. Since computer vision has the basics of image processing, artificial neural intelligence, etc. by piecing together multiple research that has already been conducted in the past, the experiments could be used as a basis for the construction of 
the simulation. Furthermore, by applying a sorting algorithm to already existing codes, the parking distances for upcoming cars could be optimized. With that in mind, future researchers can add a new feature to the parking area. Other than it being able to provide the time-in and time-out and the bill of the car, it could also provide traffic-reduced parking spaces. As the new car goes in for parking, the system could generate the different parking spots that have not yet been taken so that the driver would not have to look for a parking slot, but could immediately head for an empty one. The system would provide choices for the driver and he could immediately choose the slot so that for the next driver, that slot would be unavailable. This way, the traffic inside parking lots would be greatly reduced because the searching for spaces would be eliminated. The researchers can also acquire more training data for the neural network. Additionally, future researchers could actually create a hardware product that would be used using the code from the software. This would create raw data that could be quantized and produce actual results. If the results that were found were accurate, the implementation of this hardware can be used on parking lots around the country to reduce manual labor and create a more efficient parking ticket system.

\section{REFERENCES}

[1] E. Volna and M. Kotyrba, "Vision System for Licence Plate Recognition Based on Neural Networks." HIS Journal. Vol. 65, pp. 6-13, 2014. https://doi.org/10.1109/HIS.2013.6920470

[2] H. Rajput, T. Som, and S. Kar, "An Automated Vehicle License Plate Recognition System." Computer Systems. Vol. 48, pp. 56-61, 2015.

[3] F. Caicedo, F. Robuste, and A. Lopez-Pita. "Parking Management and Modelling of Car Park Patron Behavior in Underground Facilities." Transportation Research Record. Vol. 1956, No. 1, pp. 60-67, 2006.

[4] S. Funck, N. Mohler, and W. Oertel. "Determining Car-Park Occupancy from Single Images." Vehicles Symposium. Vol. 13, No. 2, pp. 325-328, 2004.

[5] L.S. Bartolome, A.A. Bandala, C. Lorente, and E.P. Dadios. "Vehicle Parking Inventory System Utilizing Image Recognition Through Artificial Neural Networks." IEEE Region 10 conference. Vol. 2, No. 2, pp. 1-5, 2018.

[6] Y. Qu, "Application of the Computer Vision Technology in the Image Feature Extraction." Advances in Intelligent Systems and Computing. Vol. 928, pp. 351-356, 2019.

[7] H. Zhang, "Research on the Optimizing Process of the Basic Image Processing Algorithms." Advances in Intelligent Systems and Computing. Vol. 928, pp. 212-217, 2019. https://doi.org/10.1007/978-3-030-15235-2_33

[8] Q. Liu, "Research on the Image Feature Matching Technology and its Application in the Computer Vision System." Advances in Intelligent Systems and Computing. Vol. 928, pp. 188-193, 2019.

[9] P. Caramazza, O. Moran, R. Murray-Smith, and D. Faccio, "Transmission Of Natural Scene Images Through A Multimode Fibre. Nature Communications." Nature Communications. Vol. 10, No. 1, 2019.
[10]N. O'Mahony, S. Campbell, A. Carvalho, S. Harapanahalli, G. Hernandez, L. Krpalkova, and J. Walsh, "Deep Learning vs. Traditional Computer Vision." Advances in Intelligent Systems and Computing. Vol. 943, pp. 128-144, 2019.

[11] C. Ravi, "Image Classification Using Deep Learning and Fuzzy Systems." Advances in Intelligent Systems and Computing. Vol. 941, pp. 513-520, 2019.

[12] M. Bukowski, M. Luckner, and R. Kunicki, "Estimation of Free Space on Car Park Using Computer Vision Algorithms." Advances in Intelligent Systems and Computing. Vol. 920, pp. 316-325, 2019. https://doi.org/10.1007/978-3-030-13273-6_30

[13] N. Bibi, M. Majid, H. Dawood, and P. Guo, "Automatic Parking Space Detection System.” ICMIP Journal. Vol. 2017, pp. 11-15, 2018.

[14]P. Kohli and A. Chadha, "Enabling Pedestrian Safety Using Computer Vision Techniques." Networks and Systems. Vol. 69, pp. 261-279, 2019.

[15] A. Mackay, I. Fortes, C. Santos, D. Machado, P. Barbosa, and V. Boas. "The Impact of Autonomous Vehicles' Active Feedback on Trust." Advances in Intelligent Systems and Computing. Vol. 969, pp. 342-352, 2019.

[16] A. Regester and V. Paruchuri. "Using Computer Vision Techniques for Parking Space Detection in Aerial Imagery." Advances in Intelligent Systems and Computing. Vol. 944, pp. 190-204, 2019.

[17] V. S. R. Middi, K. J. Thomas, and T. A. Harris. "Facial Keypoint Detection Using Deep Learning and Computer Vision." Advances in Intelligent Systems and Computing. Vol. 941, pp. 493-502, 2019. https://doi.org/10.1007/978-3-030-16660-1_48

[18] X. Li, H. Cui, and J. Rizzo. "Cross-Safe: A Computer Vision-Based Approach to Make All Intersection-Related Pedestrian Signals Accessible for the Visually Impaired." Advances in Intelligent Systems and Computing. Vol. 944, pp. 132-146, 2019.

[19] A. Wilkowski, I. Mykhalevych, and M. Luckner. "City Bus Monitoring Supported by Computer Vision and Machine Learning Algorithms." Advances in Intelligent Systems and Computing. Vol. 920, pp. 326-336, 2019.

[20]Ł. Sztyber. "Lane Finding for Autonomous Driving." Advances in Intelligent Systems and Computing. Vol. 920, pp. 428-444, 2019.

[21] J. Tu, "Parking Lot Guiding with IoT Way." Microelectronics Reliability. Vol. 94, pp. 19-23, 2019.

[22]B. Ajeya and S. Vincent, "Integration of Contactless Power Measuring Instruments to PLC and SCADA Through Industrial Wireless Sensor Network for EMS." Electrical Engineering. Vol. 546, pp. 279 - 292, 2019.

[23]W. Yang and P.T.I. Lam. "Evaluation of Drivers' Benefits Accruing from an Intelligent Parking Information System." Journal of Cleaner Production. Vol. 231, pp. 783 - 793, 2019.

[24] A. Capalin, M. Lim, M. Ghandehari, C. Lim, P. Glimcher, and G. Thurston. "Advancing Environmental Exposure Assessment Science to Benefit Society." Nature Communications. Vol. 10, no. 1, 2019.

[25] S.M. Dave, G.J. Joshi, K. Ravinder, and N. Gore. "Data Monitoring for the Assessment on On-Street Parking 
Aaron Don M. Africa et al., International Journal of Emerging Trends in Engineering Research, 8(2), February 2020,323 - 332

Demand in CBD Areas of Developing Countries." Transportation Research Part A: Policy and Practice. Vol. 126, pp. $152-171,2019$.

[26] P. Zhao, D. Bucher, H. Martin, and M. Raubal, "A Clustering-Based Framework for Understanding Individuals' Travel Mode Choice Behavior." Geoinformation and Cartography. Vol. 83, pp. 77-94, 2019. https://doi.org/10.1007/978-3-030-14745-7_5

[27]Z. Dzulkurnain, A.K. Mahamad, S. Saon, M.A. Ahmadon, and S. Yamaguchi, "Internet of Things (Iot) Based Traffic Management \& Routing Solution for Parking Space." Indonesian Journal of Electrical Engineering and Computer Science. Vol. 15, pp. 336-345, 2019.

[28] K. Perumal and P. Manoharan. "A Comparative Analysis of Energy-Efficient Protocols for WBAN on Heterogeneous Transceivers." Journal of Testing and Evaluation. Vol. 47, 2019.

[29] O. Urra, and S. Ilarri. "Spatial Crowdsourcing with Mobile Agents in Vehicular Networks." Vehicular Communications. Vol. 17, pp. 10-34, 2019.

[30] S. Ben Chaabene, T. Yeferny, and S. Ben Yahia. "A Roadside Unit Placement Scheme for Vehicular ad-hoc Network." Advances in Intelligent Systems and Computing. Vol. 926, pp. 619-630, 2019.

[31]L. Lou, J. Zhang, Y. Xiong, and Y. Jin. "An Improved Roadside Parking Space Occupancy Detection Method Based on Magnetic Sensors and Wireless Signal Strength.” Sensors. Vol. 19, No. 2348, 2019.

[32] T. K. Lai, A. F. Abbas, A. M. Abdu, U. U. Sheikh, M. Mokji, and K. Khalil. "Super Resolution of Car Plate Images Using Generative Adversarial Networks." Signal Processing and its Applications. Vol. 93, pp. 80-85, 2019.

[33] C. Zhang, Q. Wang, M. Wang, J. Chen, H. Liu, and S. Fu. "Intelligent Parking Management System Design from a Mobile Edge Computing (MEC) Perspective." Vehicular Technology. Vol. 35, pp. 74-95, 2018.

[34] G. T. S. Ho, Y. P. Tsang, C. H. Wu, W. H. Wong, and K. L. Choy. "A Computer Vision-Based Roadside Occupation Surveillance System for Intelligent Transport in Smart Cities." Sensors. Vol. 19, No. 2348, 2019. https://doi.org/10.3390/s19081796

[35] Y. Du, S. Yu, Q. Meng, and S. Jiang. "Allocation of Street Parking Facilities in a Capacitated Network With Equilibrium Constraints on Drivers' Travelling and Cruising for Parking." Transportation Research Part C: Emerging Technologies. Vol. 101, pp. 181-207, 2019.

[36] S. Brucal, A. Africa, and E. Dadios. "Female Voice Recognition Using Artificial Neural Networks and Matlab Voicebox Toolbox." Journal of Telecommunication, Electronic and Computer Engineering (JTEC). Vol. 10, No. 1-4, p. 133-138, 2018.

[37] R. Azad, F. Davami and B. Azad. "A Novel and Robust Method for Automatic License Plate Recognition System Based on Pattern Recognition." Advances in Computer Science: an International Journal. Vol. 2, No.4, pp. 64-70, 2013.

[38] Y. H. Chen, and H. Lee. "A Neural Network System for Two-Dimensional Feature Recognition." International
Journal of Computer Integrated Manufacturing. Vol. 11, No. 2, pp. 111-117, 1998.

[39] P. Khatri. "Car Number Plate Detection Using MATLAB and Image Processing." Circuit Digest. Vol. 4, No. 8, pp. 124-128, 2018.

[40] C. Guo, M. Rana, M. Cisse, and L. van der Maaten. "Countering Adversarial Images Using Input Transformations." Computer Vision And Pattern Recognition (CVPR). Vol. 1, No. 3, 2018.

[41]A. Africa, A. Mesina, J. Izon and B. Quitevis, "Development of a novel android controlled USB file transfer hub." Journal of Telecommunication, Electronic and Computer Engineering. Vol. 9, No. 2-8, pp. 1-5, 2017.

[42]A. Africa, "A rough set based data model for heart disease diagnostics." ARPN Journal of Engineering and Applied Sciences. Vol. 11, No. 15, pp. 9350-9357, 2016.

[43] A. Africa and C. Charleston Franklin, "Development of a cost-efficient waste bin management system with mobile monitoring and tracking." International Journal of Advanced Trends in Computer Science and Engineering. Vol. 8, No. 2, pp. 319-327, 2019. https://doi.org/10.30534/ijatcse/2019/35822019

[44] A. Africa, C. Alcantara, M. Lagula, A. Latina and C. Te, "Mobile phone graphical user interface (GUI) for appliance remote control: An SMS-based electronic appliance monitoring and control system." International Journal of Advanced Trends in Computer Science and Engineering. Vol. 8, No. 3, pp. 487-494, 2019. https://doi.org/10.30534/ijatcse/2019/23832019

[45] L. Torrizo and A. Africa, "Next-hour electrical load forecasting using an artificial neural network: Applicability in the Philippines." International Journal of Advanced Trends in Computer Science and Engineering. Vol. 8, No. 3, pp. 831-835, 2019. https://doi.org/10.30534/ijatcse/2019/77832019

[46] A. Africa, G. Ching, K. Go, R. Evidente and J. Uy, "A comprehensive study on application development software systems." International Journal of Emerging Trends in Engineering Research. Vol. 7, No. 8, pp. 99-103, 2019. https://doi.org/10.30534/ijeter/2019/03782019 